\title{
L'HISTOIRE DU SAUMON EN LOIRE
}

\author{
par R. BACHELIER, \\ Ingénieur principal des Eaux et Forêts
}

CHAPITRE IV

Suile (1)

1) I ËBI"T DE CE SHECLE A NOS JOLRS

I'industrialisation à outrance va réduire simultanément :

$1^{\circ}$ Les pêches abusives des Inscrits maritimes, du fait de l'approfondissement toujours plus grand de la Ioire à l'aval de Nantes et de l'accroissement du trafic maritime jusqu'à cette ville, devenue port de mer;

$2^{\circ}$ Les zones de frayères, du fait de l'édification de barrages beaucoup trop hauts pour ètre franchissables par les Saumons et qui comportaient des échelles à poissons inefficaces.

\section{En basse Loire}

\section{Bataille de réglementation avec l'Inscription maritime.}

Au début de ce siècle nous avons eu des renseignements assez nombreux gràce aux enquêtes auxquelles il fut procédé dans tout le bassin de la Loire pour l'application de la loi du 31 mai 1865, relative à la soumission de certains cours d'eau au "régime des échelles à poissons " prévu par cette loi.

Il est assez curieux 'de constater qu'à l'occasion de cette enquête, les concessionnaires de "sédors" (ou "polets") de la basse Loire, par une pétition en date du 13 février 1903, avaient protesté contre les pêcheurs de haute Loire (c'est-à-dire du milieu du fleuve), qui pêchaient de jour comme de nuit avec leurs filets flottants (vouillées) de plus de $400 \mathrm{~m}$ de longueur et à toute époque, alors qu'eux ne pouvaient tendre leurs sédors que le 11 janvier après la remontée des gros Saumons.

(1) Voir Bulletin franjais de Pisciculture, $\mathrm{n}^{\circ} 211$ (31 décembre 1963). 
De leur còté les dits marins-pêcheurs de haute Loire émirent aussi une pétition pour exposer leurs points de vue :

$1^{\circ}$ Les gros Saumons, qui sont déjà rendus au moins à :Ancenis lors de l'ouverture le 10 janvier, ne fraient pas en Loire puisqu'ils sont immatures.

$2^{\circ}$ Il y a 400 sédors de Cordemais à Saint-Nazaire et autant de Paimbœuf à Saint-Brévin, dont la grande majorité sont concédés à des noninscrits (cultivateurs, curés, maires, fonctionnaires, etc...). Dans 39 sédors, de Saint-Brévin à Mindin, il aurait été pêché 594 Saumons en 1901, soit plus qu'il n'en a été capturé par les 2.000 marins-pêcheurs de haute-Loire. (M. le conservateur Benardeau n'exagère donc pas quand il estime leur nombre à 1.600. Par contre ceux-ci minimisent sérieusement leurs captures, puisque l'Inscription maritime avait déclaré la capture de 30 tonnes de Saumons en 1901.)

¿.. Et firent connaître leurs désirs :

10 Suppression des sédors;

$2^{\circ}$ Ouverture de la pêche du Saumon dès le 10 décembre;

$3^{\text {o }}$ Tolérance de la pêche de nuit;

$4^{\circ}$ Surveillance de la pêche par l'administration de la Marine.

Il semble que ces pétitions contradictoires n'eurent aucune suite. Cependant le nombre, tant des sédors, que des filets dérivants alla en diminuant par suite du développement industriel de la Loire-Atlantique, qui provoquait l'embauchage de nombreux marins-pêcheurs, et de la moindre rentabilité de la pêche, du fait de la régression des remontées de Saumons et du développement de la navigation maritime à l'aval de Nantes.

C'est sans doute par suite de cette diminution des captures par les inscrits maritimes (plus que 1.500-1.600 Saumons par an) qu'il fut possible, politiquement parlant, d'obtenir la suppression du droit de pêche libre des inscrits maritimes en eau douce par deux décrets des 8 novembre et 28 décembre 1926 (1), si ce n'est pour les marins-pêcheurs en activité qui conservaient ce droit à titre viager. Ceux-ci se révélèrent être 600 , mais beaucoup d'entre eux n'avaient demandé une carte de pêche que pour ménager leurs droits.

Un arrêté du ministre de la Marine en date du 30 septembre 1934 autorisa les inscrits maritimes à pêcher avec des "chaluts" et des "trubles" de toutes dimensions et de toutes mailles dans les eaux salées, c'est-à-dire dès Cordemais. Sans doute les chaluts ne causèrent-ils des dommages qu'aux frayères des poissons d'eau salée, dont les poissons

(1) Le décret du 8 novembre 1926 avait reporté la limite de l'Inscription maritime à la limite de mer fixée par le décret du 21 février 1852 . Celui du 28 décembre 1926 a rendu le droit de pèche libre aux inscrits maritimes jusqu'à la limite des eaux salées (Cordemais), et à titre viager à ceux qui l'avaient exercé précemment jusqu'aux anciennes limites de l'Inscription maritime, soit jusqu'à Thouaré. 
plats qui, en 1892 (voir tableau page 0), remontaient encore en grande abondance au-delà de Gien. Mais il n'en fut pas, et il n'en est toujours pas de mème des "trubles ", qui sont des énormes filets en forme de poche ramassant non seulement les crevettes, à la pêche desquelles ils sont essentiellement destinés, mais aussi tous les poissons qui se laissent descendre: les Anguilles (ce qui est encore de bonne pêche), les Alosons et les Tacons!

Il est bien probable que ces trubles causent plus de dommages aux remontées futures de Saumons que tous les filets à grandes mailles pour la capture des Saumons eux-mêmes.

Les jeunes marins-pêcheurs de Nantes, sans droit de pêche à l'amont de Cordemais, obtinrent que le décret de 1926 fut partiellement rapporté par le décret du 17 juin 1938, qui remonta les limites de l'Inscription maritime a!u premier obstacle à la navigation, en l'occurence les ponts de Nantes.

Sans doute une loi du 11 mars 1941 annula "pour après la guerre" les dispositions du décret de 1938, mais cette loi fut abrogée par la loi du 24 mai 1946, si bien qu'elle ne fut jamais appliquée.

Indépendamment de cette bataille sur la limite géographique du droit de pêche pour les inscrits maritimes, il y en avait une autre sur la date d'ouverture de la pêche du Saumon.

Un décret en date du 23 novembre 1935 ayant fixé l'ouverture de la pêche du Saumon en cau salée au 25 novembre, ce décret fut annulé le 15 juillet 1938 par le Conseil d'État, car il était contraire à l'article 4 de la loi du 31 mai 1865 imposant une réglementation uniforme en eau salée et en eau douce.

\section{Achèvement des pêches abusives des Inscrits maritimes.}

Il n'empêchait que, plus ou moins officiellement, suivant les années et le poids des influences locales, les inscrits maritimes continuaient à pêcher dès le 15 novembre et à ramasser ce qu'ils trouvaient dans leurs filets.

Jusqu'en 1936, achèvement de l'approfondissement du chenal maritime à $10 \mathrm{~m}$, le nombre de leurs captures de Saumons dut osciller entre 2.000 et 6.000 et représenter la moitié de toutes les captures dans le bassin de la Loire.

De 1936 à 1949 inclus elles furent de l'ordre d'un millier, sauf en 1943,1944 et 1945 où elles furent très abondantes (ces trois années mémorables correspondirent à la diminution des pêches aux engins en 19391940 et au chant du cygne des frayères du haut Allier, qui avaient été rendues inaccessibles en 1941 par la mise en service du barrage de Poutes, à mi-hauteur des frayères à Saumons de l'Allier).

Depuis, elles sont vraiment insignifiantes pour la raison majeure qu'elles ne sont plus rentables, le prix du Saumon, si élevé soit-il, n'ayant pas plus augmenté que celui de la main-d'œuvre dans la zone industrielle de Nantes à Saint-Nazaire. 
Sans compter que le chenal maritime a encore étẻ approfondi de 2 mètres supplémentaires, et que, hors du chenal, le lit de la Loire est parsemé d'épaves depuis la dernière guerre.

En conclusion la. lutte contre les pèches abusives de Saumons par les inscrits maritimes, fut toujours une bataille d'arrière-garde.

Dès 1896, date du début de l'utilisation de la basse Loire comme canal maritime, cette lutte avait perdu beaucoup de son intérêt, et maintenant elle n'en présente vraiment plus aucun, à moins qu'elle ne soit portée sur un autre terrain (si l'on peut dire), à savoir les abus de pêche aux trubles dans l'estuaire qui, actuellement au nombre d'une quarantaine, doivent prélever beaucoup de tacons à la descente en mai-juin.

\section{En Loire moyenne}

La pêche en Loire moyenne, de Thouare à Decize, commença par augmenter au début du siècle, puisque le nombre des filets-barrages y était passé de 50 à 65 .

Mais après la dernière guerre, leur nombre se trouva réduit à 35, chacun ne comportant qu'une toue, au lieu de 2 ou 3 , sans compter 5 bouges, beaucoup moins onéreux que les filets-barrages, mais aussi bien moins efficaces.

Comme suite à un væu formulé par la Commission des migrateurs, en 1949, les adjudicataires, à l'occasion des nouvelles adjudications, ne furent autorisés à utiliser des filets-barrages que dans les lots où un tel engin était demeuré en service.

De ce fait, aujourd'hui leur nombre est réduit à 25 filets-barrages et 3 bouges, et depuis ces deux dernières années, les captures de Saumons à la ligne sont même plus nombreuses que celles réalisées à l'aide de ces engins.

Quelques exemples depuis 1949 :

Inscrits barrages - Lignes Total

1950 (bonne annice) :

Saumons d'hiver ........ $220 \quad 1.150$

Saumons de printemps $\ldots \quad 0 \quad 200$

$383(1) \quad 1.753$

1951 (très mauvaise) :

Saumons d'hiver ........ 35

265

104

Saumons de printemps ... 0

(1) Dont 250 au pied du barrage des Laurins en reconstruction sur le bas Allier alors que normalement les Saumons ne se pêchent pas à la ligne à l'aval de ce barrage. 


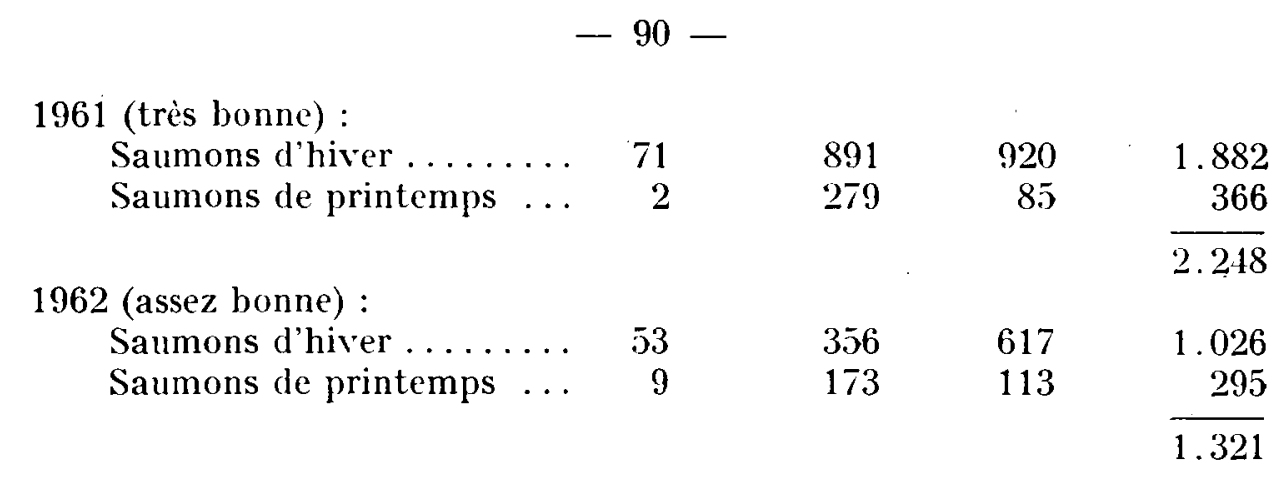

Or la pêche du Saumon à la ligne n'existait pas au début du siècle et entre les deux guerres (donc avant la construction du barrage du Poutes). dans le parcours le plus fréquenté par les pêcheurs à la ligne, à savoir l'Allier, à hauteur de Brioude, il n'était capturé annuellement que 100 à 200 Saumons.

Mais une menace autrement grave que les filets-barrages pèse sur la Loire moyenne, il s'agit de la centrale nucléaire de Chinon.

Comme au début du siècle dernier, les barrages de Châtellerault et de Decize, cette nouvelle application des progrès mécaniques parut devoir être sans effet sur les remontées de Saumons. Puis, un beau jour, on apprit qu'il était nécessaire d'élever un petit barrage rustique à travers la Loire, afin d'assurer un apport d'eau suffisant à l'usine pour le refroidissement des piles au cours des assecs d'été.

Et à l'occasion de la réception de la passe à poissons exigée à travers ce barrage, il fut révélé d'une part, que des poissons étaient détruits dans les grilles tournantes protégeant la prise d'eau de refroidissement de la première pile, et, d'autre part, que lorsque toutes les piles prévues seraient en marche, celles-ci auraient besoin d'un débit d'eau de $50 \mathrm{~m}^{3} /$ seconde $! .$. .

Or les débits d'étiage de la Loire tombent assez souvent au-dessous de ce chiffre. Sans doute, lors de ceux-ci, il n'y a normalement pas de descente de tacons. Mais une crue locale, en mars ou avril dans une zone de frayères, peut déclencher une descente de tacons qui dériveront à hauteur de la centrale de Chinon, alors que le débit de la Loire pourra n'être que de quelques centaines de mètres cubes par seconde. Quelle sera la proportion des tacons entraînés vers les grilles tournantes?

Ces grilles tournantes étant aussi gigantesques et perfectionnées qu'il est possible de l'imaginer, aucune mesure de sauvegarde n'est envisageable de ce côté.

Faute de mieux, il a donc été demandé à la centrale nucléaire de Chinon d'installer un écran électrique à l'entrée de son canal de prise d'eau, mais celui-ci a 120 mètres de largeur et sa profondeur peut varier de 2 mètres à 10 mètres suivant les crues.

Le problème qui était posé par les barrages de Châtellerault et de Decize était bien simple à résoudre à côté de celui-ci. Car non seulement la constitution d'un écran électrique dans une section de $120 \times 10$ $=1.200 \mathrm{~m}^{2}$ n'est pas chose simple, mais l'efficacité d'un tel écran vis-àvis des tacons descendants (smolts) n'est pas encore bien définie malgré les nombreuses recherches américaines, anglaises et allemandes à ce sujet. 


\section{En Loire supérieure}

\section{Le barrage de La Vourdiat}

La raréfaction des remontées de Saumons en Loire supérieure, qui avait été causée insidieusement par les barrages de navigation de Decize et de Roanne (de plus en plus perfectionnés mécaniquement et, de ce fait, de plus en plus difficiles à franchir), puis partiellement, mais brusquement, par le barrage de Brive-Charensac, en 1898, fut parachevée par la construction du barrage-usinier de La Vourdiat, de 5 mètres de hauteur, au Saut du Pinay $(30 \mathrm{~km}$ à l'amont de Roanne, donc en aval de la zone à frayères), en 1909 .

Pendant 5 années successives, ce barrage arrêta et causa la mort de plusieurs centaines de Saumons, puis encore, en 1921 , de 250 qui avaient dû naître en Loire supérieure à la suite d'une crue ayant permis à un certain nombre de Saumons d'atteindre la zone de frayères, en 1916. Par la suite il ne fut question que de l'observation de quelques Saumons isolés en I.oire supérieure les années de grandes crues. Une échelle Caméré avait bien été dressée contre ce barrage, mais comme toutes les échelles de ce type, celle-ci ne fut jamais utilisée par quelque poisson que ce fut avant d'être emportée par une crue.

En 1927, l'inspecteur des Eaux et Forêts Roy fit aménager une passe rustique dans la rive droite, et bien que cette passe débouchât à $200 \mathrm{~m}$ à l'aval du barrage, presque chaque année des Saumons devaient l'utiliser, car il en était observé quelques-uns assez régulièrement jusqu'à La Voute (Loire), au pied du barrage du Gouffre Bernard, de $4 \mathrm{~m}$ de hauteur qui avait été construit en 1932.

A l'occasion d'une crue qui avait provoqué une petite brèche dans le barrage de La Vourdiat, neuf Saumons furent encore vus franchir cette brèche le jour de Pâques 1952.

\section{Tentative de réacclimatation}

Cette même année 1952, fut ouverte l'enquête préalable à la concession du grand barrage de Grangent, sur la Loire, à hauteur de Saint-Étienne. La retenue de ce barrage, haut de $50 \mathrm{~m}$, devait submerger $20 \mathrm{~km}$ de Loire qui, jadis, y constituaient la partie aval de la zone à frayères de Saumons.

Mème compte tenu de l'observation toute récente de la remontée de 9 saumons à I a Vourdiat, il ne pouvait pas être imposé au concessionnaire d'investir plusieurs dizaines de millions de francs dans la construction d'une passe à Saumons qui ne servirait peut-être jamais, mais il lui fut demandé de ménager la possibilité de construire un tel ouvrage si la restauration des remontées de Saumons en Loire s'avérait possible.

Ayant disposé d'œufs de Saumons de la Baltique à partir de 1953, chaque année plusieurs dizaines de milliers de ces œufs furent affectés à l'ensemencement de la Loire supérieure au moyen de boîtes Vibert. 
Puis les barrages responsables de la quasi-disparition des Saumons en Loire supérieure furent vaincus successivement en partant de l'aval :

Un accord fut passé avec le Service de la Navigation pour que le barrage de Decize fut ouvert chaque dimanche en mars, avril et mai.

La Fédération de Pêche et de Pisciculture de la Loire, avec l'aide du Conseil supérieur de la Pèche, construisit deux échelles à poissons dans le barrage de Roanne, dont l'une spécialement pour les Saumons.

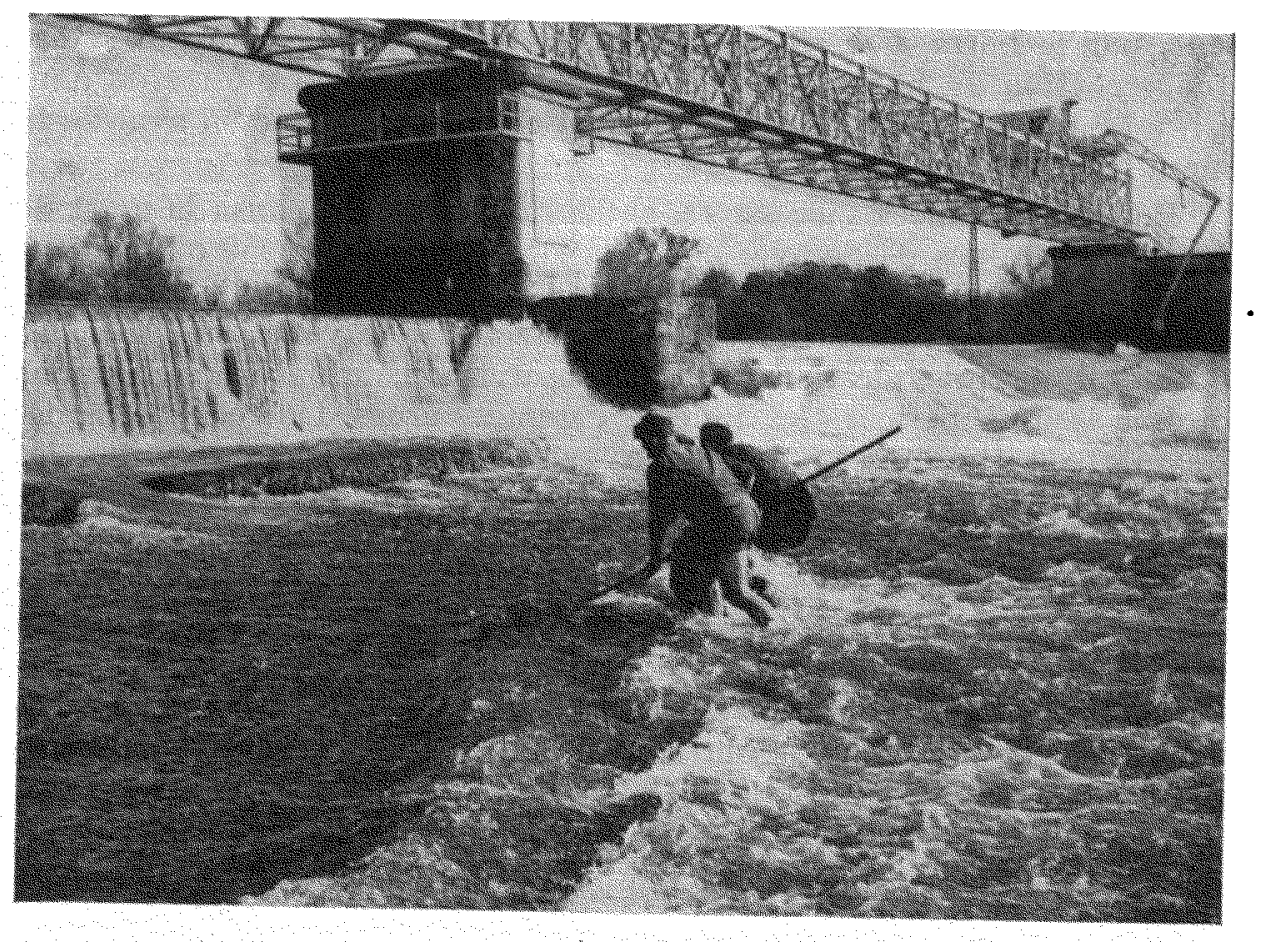

Fig. 3 - Barrage de Decize, de 1932-1933, par crue moyenne. (Pèche de destruction de Hotus.)

Les Saumons ne peuvent franchir lu travé mon abuissice (à gauche), faute de pouvoir effectuer un double saut, ni la travée abaissée (à droite) parce que le courant y est trop rapide.

Il fut obtenu du Service de l'Exploitation d'E. D. F. qu'il aménageàt une passe à Saumons fort simple dans le barrage de La Vourdiat.

Mais lorsque les Eaux et Forèts furent consultées par le Service de l'Équipement d'E. D. F., en 1957, au sujet des mesures à prendre dans le barrage de Grangent afin de pouvoir y installer éventuellement une écluse à poissons, il était déjà trop tard, car ce barrage fort important était pratiquement achevé.

Ce service, comme tous les constructeurs de barrages et même, jadis, ceux des échelles à poissons, n'avait pas pensé que la condition primordiale pour l'efficacité d'une passe à Saumons, est que celle-ci soit alimentée 
par un débit d'au moins $2 \mathrm{~m}^{3} / \mathrm{s}$ dans un fleuve comme la Loire. Le gros œuvre en béton armé du harrage étant achevé, il n'était plus possible d'y ouvrir un pertuis pour assurer le passage d'un tel débit.

A vrai dire, du jour où l'aménagement hydroélectrique de la Loire supérieure avait été décidé, à l'instigation notamment de la Ville de Paris qui n'a jamais renoncé au projet de captage des eaux du Val de Loire, il n'y avait plus guère d'espoir de restaurer les remontées de saumons en Loire supérieure. En effet, s'il est facilement imaginable de faire remonter par des Saumons des barrages de $50 \mathrm{~m}$ de hauteur au moyen d'écluses ou d'ascenseurs, le problème de la descente des tacons à travers une retenue de $20 \mathrm{~km}$ de longueur, et un barrage si élevé, n'est pas encore résolu, même aux U.S. A.

Sans compter qu'il est probable que la Ville de Paris, avec l'aide d'E. D. F., réalisera son projet du barrage de Villerest, à l'amont immédiat de Roanne, qui, lui, aurait une retenue de près de $40 \mathrm{~km}$ de longueur!

En conclusion, il n'y a plus aucun espoir de voir un jour des Saumons remonter dans la I coire supérieure.

\section{En Vienne}

Les "saumonières" ayant été interdites en 1890, les propriétaires des barrages ne durent plus être tentés de rendre ceux-ci plus difficilement franchissables pour augmenter leurs captures de Saumons.

Mais le progrès mécanique était ven:ı prendre le relai du braconnage organisé pour s'opposer aux remontées de Saumons. Sans avoir à demander une autorisation quelconque, les propriétaires pouvaient, aux termes de la loi du 15 avril 1898, sur le Régime des Eaux, remplacer leurs "rouets" par des turbines capables d'absorber des débits beaucoup plus importants, si bien que les déversements sur les chaussées, qui seuls permettaient aux Saumons de remonter, se firent de plus en plus rares et plus faibles.

A ce sujet, cette loi du 15 avril 1898 ignorait tellement les sujetions piscicoles que les cahiers des charges pris pour son application exigeaient l'ouverture partielle ou totale des vannes sitôt que la chaussée risquait de déverser.

Heureusement, cette clause ne fut jamais respectée, car le Saumon a beau nager avec une très grande vitesse, il a été reconnu qu'il était moins rapide que l'eau se déversant sur $80 \mathrm{~cm}$ de hauteur. C'est dire que, même à travers le pertuis d'une vanne grande ouverte, il ne peut remonter que si la dénivellation entre les deux biefs est inférieure à $80 \mathrm{~cm}$, tandis qu'en sautant hors de l'eau sur le front de la chaussée déversante, il peut remonter une dénivellation de $2 \mathrm{~m}$, hauteur qui était rarement atteinte par les barrages-chaussées du $\mathrm{xrx}^{\mathrm{e}}$ siècle.

Ces perfectionnements des petits barrages comme obstacles aux remontées de Saumons sont passés inaperçus parce que ceux-ci ne furent apportés que progressivement (en plusieurs décennies) et ne jouaient à plein que lors des aznées sèches. 
Nul ne se plaignait qu'une certaine année il ait pu être pèché (ou simplement ramassé) quelques Saumons à l'aval d'une chute modernisée.

Mais en supposant que, lors d'une année sèche ou mème seulement peu humide, chaque chute modernisée arrêtait (et stérilisait donc) $20 \%$ des Saumons de remontée, cette remontée était réduite de moitié à l'amont de la $3^{\mathrm{e}}$ chute et des $4 / 5$ à l'amont de la $7^{\mathrm{e}}$, indépendamment des captures par les pêcheurs et autres déprédateurs (braconniers, loutres, hérons, etc...). Or, dès le début de notre siècle, les Saumons nés dans le bassin de la Vienne rencontraient déjà beaucoup plus de 7 chutes équipées de turbines avant d'atteindre leurs frayères d'origine.

Compte tenu de ce que les Saumons remontent frayer dans leurs frayères d'origine à quelques centaines de mètres près, on conçoit que des frayères supérieures, qui n'ont pas pu être atteintes une certaine année sèche, seront désertées l'année, fut-elle humide, où la génération non née au cours de l'année sèche aurait dû se reproduire (gros Saumons : 5 ans après; moyens saumons : 4 ans; et petits Saumons : 3 ans).

Cette régression insidieuse et progressive dans le temps le fut donc aussi dans l'espace, en ce sens qu'elle commença à condamner à la stérilité, faute de géniteurs, les frayères les plus en amont. Et il s'agissait justement des meilleures frayères parce que les eaux les baignant étaient toujours assez riches en oxygène pour les tacons, même lors des étés chauds.

Les autorités étaient bien disposées vis-à-vis des Saumons, puisqu'elles obtinrent, non sans mal d'ailleurs, la soumission du bassin de la Vienne au régime des échelles à poissons en 1904, mais malheureusement elles n'avaient pas suffisamment de notions de pisciculture pour conjurer le mal auquel, pourtant, il pouvait encore être remédié.

Parce qu'il ne remontait plus que quelques Saumons d'une année à l'autre, elles laissèrent construire :

En 1900-1901 .... Le barrage de Chăteauponsac ( $5 \mathrm{~m}$ de hauteur), sur la Gartempe.

En 1900-1901 .... Le barrage de Bonavaux (10 m), à Glenic (1), sur la Creuse.

En $1902 \ldots \ldots \ldots$ Le barrage de Maureix (3 m), sur la Bas-Taurion.

Dans les archives relatives à l'exhaussement de ce dernier barrage, en 1902, il est signalé que dans une braie, en 1890, il y aurait été capturé 120 livres de tacons en une nuit! (Et je ferai remarquer qu'il ne pouvait s'agir que d'une braie à anguilles pour la pêche d'avalaison à l'automne, or les tacons ne descendent qu'au printemps... Nul doute donc que cette braie était aussi utilisée à la capture des tacons et que la relation de la capture de tacons de 1890 n'ait été signalée qu'en raison de son importance - en effet plusieurs milliers !)

Puis, en 1907-1909, le barrage de Ia Roche-Bat-L'Aigle, de $5,50 \mathrm{~m}$ de hauteur, à Gargilesse, sur la Creuse, dont désormais toutes les frayères à Saumons furent condamnćes à la stérilité.

(1) Glenic : nom vernaculaire dutacon : 
Par conséquent, déjà avant la première Grande Guerre, les remontées de Saumons étaient excessivement clairsemées dans tout le bassin de la Vienne, et ceux-ci ne disposaient plus que de 310 ha des 760 ha d'eau qui étaient capables de produire des tacons arant la Rérolution.

En fait, il ne devait plus guère remonter que quelques centaines de Saumons dans l'axe Creuse-Gartempe après avoir payé un lourd tribut au barrage de La Haye-Descartes, et moins encore dans la Vienne moyenne et ses petits afmuents après hécatombe au barrage de Chàtellerault.

La construction du barrage du Bec-des-Deux-Eaux (ou de MaisonsRouges), de $5 \mathrm{~m}$ de hauteur, en 1918-1920, à l'aval immédiat du confluent de la Creuse et de la Vienne, sonna le glas du Saumon dans tout le bassin de la Vienne, si ce n'est un retour très temporaire en 1922-1923 à la suite de la destruction partielle du barrage par une crue fin 1922.

Les Eaux et Forêts y avaient bien fait construire une échelle à poissons d'un type qui avait donné toute satisfaction au maintien des remontées de Saumons dans la Meuse. Mais le débit de cette échelle était insuffisant comparativement aux $100 \mathrm{~m}^{3} / \mathrm{s}$ turbinés nuit et jour par l'usine, et son pied, sans être tout à fait mal situé, ne l'était pourtant pas suffisamment bien pour attirer une proportion importante des Saumons bloqués au pied du barrage.

A remarquer qu'après quelques années de pèches exceptionnelles (et pour cause !) an pied du barrage, il ne fut plus jamais revu de Saumons dans les $70 \mathrm{~km}$ de Vienne qui s'étendent entre son confluent avec la Loire et ce barrage. Dès cette époque, sans attendre les résultats des observations de marquage à l'étranger, on devait déjà savoir, de ce fait et d'une manière certaine, que les Saumons ne remontaient que dans les rivières où ils étaient nés.

Non seulement les pêcheurs de la basse Vienne l'apprirent à leur dépens, mais même les pêcheurs de Loire, à l'aval du confluent de la Vienne. Du jour où il n'y eut plus de Saumons à remonter en Vienne, les pêcheurs de Loire, loin à l'aval du confluent de la Vienne, renoncèrent à tendre leurs filcts-barrages à partir de la rive gauche, car ils n'y pêchaient presque plus de Saumons. A vrai dire, ce déséquilibre des remontées de Saumons entre les deux côtés de la Loire à l'aval du confluent de la Vienne n’a duré qu'une dizaine d'années.

En même temps que le barrage du Bec-des-Deux-Laux, il avait été construit à $75 \mathrm{~km}$ à son amont, sur la Vienne, le barrage de Ia Roche, à L'Isle-Jourdain, de $12 \mathrm{~m}$ de hauteur, pour lequel les Eaux et Forêts firent construire la plus grande échelle à poissons qui ait jamais existé en France. Du même type et pas mieux située que celle du barrage du Becdes-I)eux-Eaux, elle fit couler beaucoup d'encre et ne livra passage qu'à quelques Saumons de ceux qui avaient pu, à l'occasion de la grande crue de fin 1922, franchir le barrage du Bec-des-Deux-Eaux (partiellement emporté) et le barrage de Châtellerault (dont la hauteur a d'ailleurs été portée à $3 \mathrm{~m}$ en 1928).

Si bien que le barrage de Chardes, de $10 \mathrm{~m}$ de hauteur, qui fut construit à $1, \bar{j} \mathrm{~km}$ à l'aral de celui de La Roche, en 1921-1926, et le barrage de Jousseau, encore de $10 \mathrm{~m}$ de hauteur à $10 \mathrm{~km}$ à l'amont, en 1926-1928, ne comportèrent ni l'un ni l'autre d'échelle à poissons. 


\section{Réouverture de la Gartempe.}

Malgré ce triple verrouillage total de la Vienne supérieure, qu'elles ignoraient peut-être, plusieurs personnalités influentes du département de la Vienne obtinrent, en 1949, le vote de crédits du Conseil général de la Vienne d'abord, puis des Conseils généraux de l'Indre-et-Loire et du Maine-et-Loire et du Conseil supérieur de la Pêche, pour édifier dans le barrage du Bec-des-Deux-Eaux une échelle du type qui avait fait ses preuves vis-à-vis des saumons des Gaves et qui débitait $1.000 \mathrm{l} / \mathrm{s}$ au lieu des $300 \mathrm{l} / \mathrm{s}$ de l'échelle primitive.

En 1950-1951, cette échelle fut construite en tunnel dans la rive gauche, sous l'entrée de la centrale hydroélectrique, de sorte qu'elle devait permettre la remontée des Saumons appelés par les restitutions des turbines, ce qui ne devait pas manquer de se produire au moins après les crues, lorsque les déversoirs sont taris.

Une nasse de contrôle fut placée en tête de l'échelle en avril 1952, et il y fut capturé du 16 avril au 17 mai 1952, 819 Lamproies, 251 Hotus, 73 Soffies, 48 Aloses, 47 Barbeaux et 6 Chevennes.

En même temps que cette échelle à poissons était construite, les Papeteries de Balesmes étaient invitées à moderniser et à prolonger l'échelle à poissons de leur barrage de La Haye-Descartes sur la Creuse, qui avait été exhaussé de 0,25 en 1924 . Ce qui fut fait en 1952.

D'autre part, dès que l'ouverture de ces deux barrages avait été certaine en 1951, des œufs de Saumons furent immergés en boîtes Vivert dans les affluents de la Gartempe à l'aval du barrage de Châteauponsac, ces cours d'eau devant ètre accessibles aux Saumons qui auraient franchi les dits barrages.

Les enfouissements d'œufs de Saumons originaires des Gaves portèrent sur : 50.000 en $1951,80.000$ en $1952,35.000$ en 1953 et 200.000 en 1954, puis, faute de suffisamment d'œufs de Saumons des Gaves, ces ensemencements furent poursuivis avec des oufs de Saumons de la Baltique.

Un Saumon de $3 \mathrm{~kg}$ fut pêché en basse Vienne, en novembre 1954 , puis 3 gros Saumons furent vus successivement au barrage du Bec-desDeux-Eaux en mai 1958, et au barrage de Chome, a hauteur de Bellac, au mois de juillet suivant.

A la suite de cette observation, E. D. F., propriétaire du barrage du Bec-des-Deux-Eaux, fut priée de construire l'échelle à poissons qui pouvait être imposée à cette chute en raison de l'exhaussement de $0,40 \mathrm{~m}$ qui y avait été apporté depuis le décret de concession du 2 février 1917.

Cette échelle fut construite immédiatement à l'extrémité droite de la chaussée-déversoir pour permettre la remontée des Saumons par hautes eaux (dans ce cas, en effet, les débits à travers la chute sont plus importants le long de la rive droite que le long de la rive gauche, où se trouve l'usine et la première échelle à poissons qui a été établie pour faciliter les remontées de Saumons lors des basses eaux).

De même le permissionnaire du barrage de Chome fut mis en demeure 
d'exécuter l'échelle à poissons qui lui avait été imposée par son règlement d'eau du 11 décembre 1921, mais qui n'avait pas été exigée à l'époque, en raison de la disparition totale des Saumons. Cette échelle à poissons fut réalisée sous forme d'écluse à poissons en 1960-1962.

\section{Echec de l'acclimatation du Saumon de la Baltique.}

Depuis 1958, il n'a pas été revu de Saumon dans la Gartempe malgré la poursuite des ensemencements en cufs de Saumons de la Baltique depuis $19 \overline{5} 4$.

En fait, alors que depuis $19 \bar{a} 4$ plusieurs centaines de milliers d'ceufs de Saumons et de grosses Truites de mer de la Baltique ont été enfouis chaque année dans de nombreux fleuves de l'Atlantique et de la Manche, on a constaté, dans la quasi-totalité des estuaires, des remontées de Salmonides de $1 / 2$ à $2 \mathrm{~kg}$ généralement en juillet ou août, mais jamais de gros Saumons pesant de 8 à $10 \mathrm{~kg}$ comme ceux de la Baltique.

Sans doute en basse Loire, il a été signalé des captures de petits Saumons plus trapus et à tête plus courte que les Saumons habituels, en 1956,1957 et 1958 (il se peut qu'il en soit toujours ainsi, mais que ces Saumons trapus ne soient plus remarqués, parce que, eux aussi sont devenus "habituels"). Au début, on aurait pu croire que ceux-ci étaient des Saumons polonais plus ou moins acclimatés, car leur poids moyen avait augmenté d'une année à l'autre : $2,5 \mathrm{~kg}, 3,5 \mathrm{~kg}$ et $4,5 \mathrm{~kg}$. Mais aucun Saumon de ces remontées ne fut observé dans le bassin de la Vienne, et il est donc plus probable qu'il s'agissait de Saumons atlantiques canadiens provenant des alevinages effectués par l'Association protectrice du Saumon.

A la suite d'un voyage en Pologne, à la fin de 1960, une explication de cet échec d'acclimatation m'est apparue.

Saumons de la Baltique et Truites de mer de la Baltique présentent, quant à leurs poids ou à l'éloignement de leurs frayères, des variètés comparables. Une petite minorité de l'une et l'autre espèce ne pèse que 4 à $6 \mathrm{~kg}$, alors que leur poids normal est de 8 à $10 \mathrm{~kg}$. Chacune a des zones de fraie distinctes, celles-ci pouvant se trouver à quelques dizaines de kilomètres de la mer, ou à $1.000 \mathrm{~km}$ (Truites du Dunajec et Saumons de la Sola, affluents de la Vistule, dans les Karpathes).

Ni l'une ni l'autre ne s'échappe de la mer Baltique, en fait mer presque intérieure, sans marée ni profondeur, dont les eaux, fort peu salées, sont excessivement riches en nourritures. Mais, comme en eaux douces, elles $\mathrm{y}$ ont chacune leur territoire, les truites de mer se réservant exclusivement les hauts-fonds côtiers jusqu'à $10 \mathrm{~m}$ de profondeur seulement.

La grosseur de ces poissons s'explique facilement par la richesse biogénique remarquable de la mer Baltique. Par contre, on conçoit facilement que leur progéniture transplantée sur les côtes de la Manche et surtout de l'Atlantique, demeure chétive en raison de la pauvreté biogénique relative de leurs eaux et de l'exiguïté des hauts-fonds de moins de $10 \mathrm{~m}$ pour les Truites et de moins de $100 \mathrm{~m}$ pour les Saumons. 
Sans compter que ces races, en fait fort peu maritimes, ne savent certainement pas emprunter la route des smolts des Gaves vers l'océan Arctique, ni même peut-être la Manche vers la mer du Nord.

Par contre, les Saumons canadiens étant des poissons des océans Atlantique et Arctique, il est concevable que leur descendance transplantée sur les côtes Est de l'Atlantique sache y retrouver leur espace vital héréditaire.

\section{Échec du transfert de géniteurs de Loire-Allier.}

Pour augmenter les chances de réacclimatation du Saumon dans la Gartempe, en février 1958, des Saumons furent achetés vivants à des pêcheurs au filet-barrage d'Indre-et-Loire et transportés par les soins de la Fédération de pêche et de pisciculture d'Indre-et-I oire dans la haute Gartempe.

Mais, là encore, cette opération a été un échec, car plusieurs des Saumons ainsi immergés ont été retrouvés morts dans des grilles de moulin de la basse Gartempe quelques semaines ou mois après leur déversement.

Nul doute que ces Saumons morts avaient tenté de retrouver leur chemin pour l'Allier, et que, s'il y en eut qui survécurent, ceux-ci y retournèrent via la Loire.

\section{Programme en cours.}

L'ensemencement a été repris en décembre 1961 avec des oufs de Saumons de l'Allier et il sera poursuivi soit avec de ces oeufs, soit avec des œufs de Saumons des Gaves suivant les possibilités.

En outre, des demandes de concessions de chutes sur la moyenne Gartempe ayant été déposés, cette fois il a été demandé l'aménagement d'écluses à Saumons à gros débit dans chaque barrage et les plans en ont déjà été établis.

Lorsque des remontées de Saumons auront été obtenues en moyenne Gartempe, il y aura lieu de vaincre le barrage de Châtrauponsac, afin que ces Saumons réacclimatés disposent des 80 ha d'eau des zones à frayères de la haute Gartempe, du siècle dernier.

Par ailleurs, il n'y a plus aucun espoir dans le bassin de la Vienne. Nous avons vu qu'un triple verrouillage parfait existait en Vienne moyenne depuis 1925 (Chardes, La Roche et Jousseau), et vaincre celuici à grands frais ne serait d'aucune utilité par suite des aménagements hydró̀lectriques d'l. D. F. intervenus depuis à leur amont.

Toutes les eaux du bassin supérieur de la IJaute-Vienne sont dérirées dans la Naulde qui a été transformée en une suite ininterrompue de retenues et le Taurion a subi la même transformation il y a déjà 30 ans. 
Les 80 ha de zones a frayères de la Gartempe seront bien peu à còté des 760 qui existaient jadis dans le hassin de la Vienne, mais nous verrons plus loin qu'il n'en subsiste pratiquement plus que 200 ha dans l'Allier. S'il remontait dans la Gartempe un tiers des Saumons de l'Allier, ce serait tout de mème un succès.

\section{En Allier}

Dans le bassin de l'Allier, tout aurait pu se dérouler comme dans la Vienne, d'autant plus que les services avaient confiance dans l'efficacité des échelles à poissons qui leur étaient conseillés par l'administration centrale.

Heureusement, ni l'État, ni aucune société n'eut liidée d'y aménager une chute hydroélectrique à l'aval de la totalité des frayères, comme cela avait été le cas dans la Loire, la Vienne et la Creuse.

Cependant, après l'édification du barrage de la Jonchère, à SaintÉtienne-du-Vigan, en 1895-1896, qui stérilisa les plus hautes frayères de l'Allier, donc les meilleurs, de nouveaux obstacles furent encore opposés aux remontées de Saumons :

$1^{\circ}$ En 1904, la chute de Sauviat a été aménagée sur la Dore $(6 \mathrm{~km}$ amont de Courpierre), exactement à l'extrémité aval des zones de frayères de cette rivière. Le barrage de cette chute, de 1,50 $\mathrm{m}$ de hauteur, était franchissable par les saumons, avec ou sans échelle d'ailleurs, mais, première chute modernisée, celle-ci comportait une dérivation ne laissant dans la Dore, sur une longueur de $2,500 \mathrm{~km}$, qu'un débit de $100 \mathrm{l} / \mathrm{s}$ hors des crues. On peut imaginer à quel genre de pêche ce barrage a donné lieu à l'époque, et pendant longtemps d'ailleurs, car en 1936, lors de l'enquête piscicole nationale, il était signalé encore quelques remontées de Saumons jusqu'à Ambert.

Depuis cette époque, ce barrage a été exhaussé à deux reprises et, de ce fait, rendu totalement infranchissable.

$2^{\circ}$ Vers 1906-1907, l'ancien barrage dénommé de Lempdes sur l'Allagnon, et dit aujourd'hui du Grand-Pont (aval immédiat du pont de la R. N. 9), fut exhaussé de 2 à $3 \mathrm{~m}$ et le barrage dénommé actuellement de Lempdes (à $2 \mathrm{~km}$ plus à l'amont) fut construit avec une hauteur de $4,20 \mathrm{~m}$.

Sans doute ces barrages furent agrémentés d'ouvrages dénommés "échelles à poissons ", mais jamais un Saumon ne s'y engagea et les 130 ha d'eau capables de produire des tacons dans l'Allagnon devinrent définitivement stériles.

$3^{\circ}$ En 1927 ce fut le Chapeauroux, qui fut interdit aux Saumons par la construction d'une chute hydroélectrique privée, dont le barrage comportait un ouvrage qui avait le nom "d'echelle it poissons", et dont les Saumons ignoraient l'usage.

$4^{0}$ Enfin, malgré de très fortes oppositions, la chute de PoutesMonistrol, prévue dès 1930, fut aménagée en 1939-1941 pour couvrir 
les besoins énergétiques de la nation en guerre et elle coupa tout le haut Allier aux Saumons.

Sans doute le barrage de Poutes, haut de $16 \mathrm{~m}$, avait été pourvu d'une échelle à Saumons du type ayant donné satisfaction dans les Gaves, mais cette échelle ne permettait la remontée que sur $7 \mathrm{~m}$ de hauteur, si bien qu'elle ne pouvait être mise en service qu'après que le niveau de la retenue ait été abaissé de $9 \mathrm{~m}$ !

Cette charge était d'autant plus lourde pour le concessionnaire que les Saumons ne remontaient au pied du barrage de Poutes, situé à près de $10 \mathrm{~km}$ à l'amont de la restitution de l'usine de Monistrol, que lors des crues qui provoquaient un déversement du dit barrage. C'est donc justement après son remplissage par une crue qu'il fallait le vidanger aux $3 / 4$. Malgré cette grande perte d'énergie, le concessionnaire mettait l'échelle en service chaque fois que les gardes avaient observé des Saumons au pied du barrage, ce qui se produisait assez régulièrement après la crue printanière d'avril ou mai.

Par contre, sauf en 1941 et 1948, il ne fut jamais observé de descente de tacons dans l'Allier au-dessus du barrage de Poutes, si bien que la " passe à tacons " aménagée dans le dit barrage ne fut jamais mise en service depuis 1943 .

Vers 1950 , la solution suivante fut proposée à l'E. I. F. : celle-ci installerait un piège à Saumons dans le bas de l'échelle à Saumons et, en entr'ouvrant la vanne de fermeture de celle-ci, elle y laisserait s'écouler un débit de l'ordre de $1 / 2 \mathrm{~m}^{3} / \mathrm{s}$ chaque fois et tant qu'il y aurait des Saumons au pied du barrage. De la sorte E. D. F. n'aurait plus à gaspiller sa réserve d'eau pour mettre l'échelle à poissons en service, et l'administration s'emparant des Saumons dans le piège (normalement en avril ou mai), en connaîtrait le nombre et pourrait les mettre à passer l'été en lieu sûr, par exemple dans les retenues à eaux fraîches toutes voisines sur l'Ance-du-Sud.

Ce n'est qu'en 1958 qu'E. I. F. réalisa ce piège, et jusqu'ici celui-ci n'a permis que la capture d'un Saumon, faute de remontées au barrage de Poutes.

En fait, pour réamorcer ces remontées, il faudrait procéder à un ensemencement massif en œufs de Saumon du haut Allier, au moins une année, afin d'obtenir une descente de tacons suffisamment importante pour qu'elle soit visible et permettre la mise en service de la "passe à tacons" du barrage.

$5^{\mathrm{o}}$ Il y eut encore une alerte en 1949 , lors de la réfection et de la modernisation du vieux barrage des Laurins sur le bas Allier, dont il a été parlé au début de cet article (p. 89).

Indépendamment de ces nouveaux obstacles dressés dans le bassin de l'Allier depuis le début de ce siècle, deux faits nouveaux, liés d'ailleurs partiellement l'un à l'autre, y eurent lieu : la suppression de la pêche aux engins à l'amont d'Issoire et la pullulation du Hotu au moins jusqu'à Brioude.

Sous la pression des pêcheurs à la ligne, l'administration des Eaux 


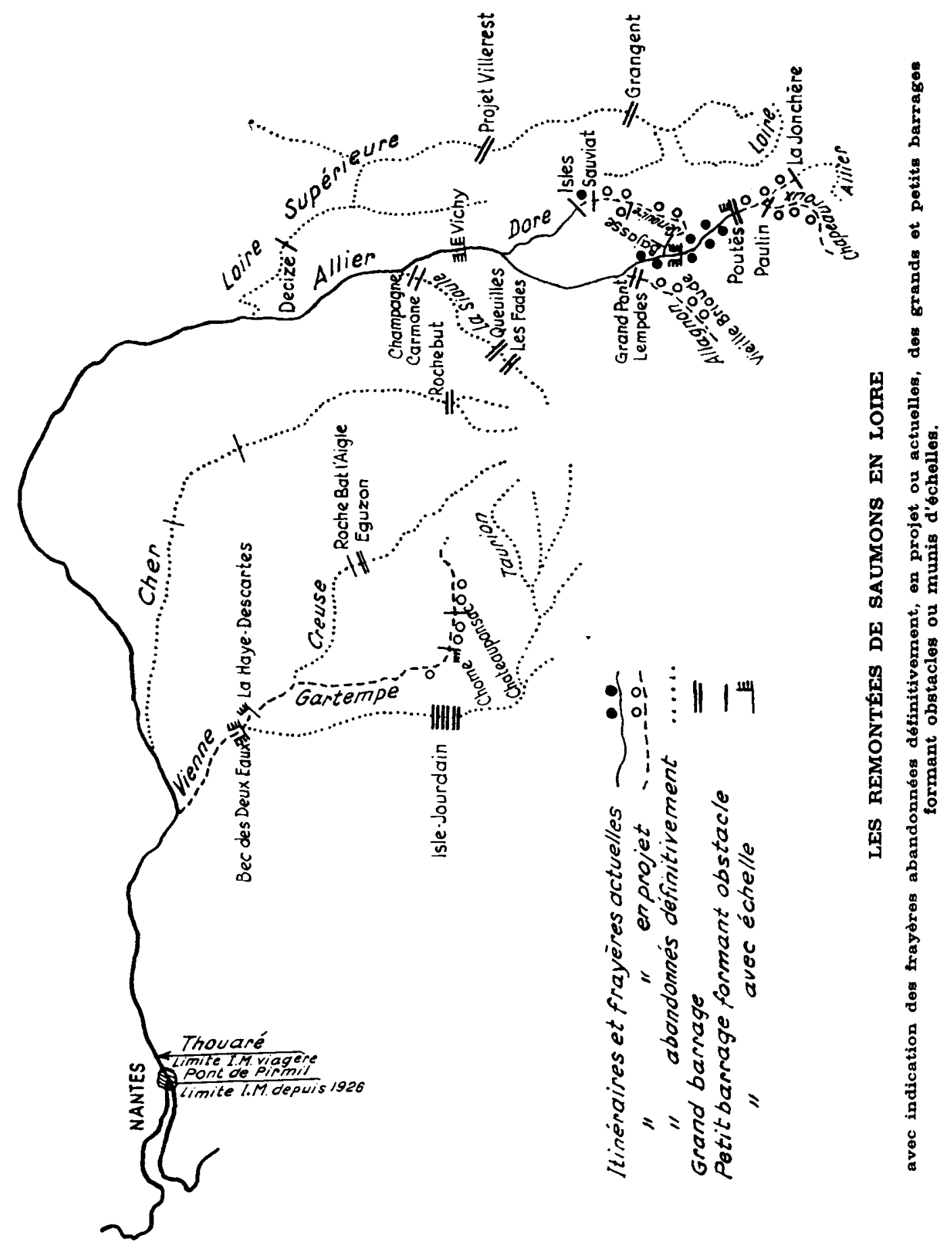


et Forèts n'a plus adjugé la pèche aux engins à l'amont d'Issoire. Et pourtant, dès avant ce retrait de la pèche aux engins, plusieurs pêcheurs professionnels avaient renoncé à l'utilisation de leurs engins pour pêcher le Saumon au lancer, ce qui semblait bien prouver que la pèche aux engins, bien à l'aval des zones de fravères il est rrai, n’était pas spécialement meurtrière pour les Saumons.

Par contre, il est certain que cet abandon de la pèche aux engins a été favorable à l'envahissement de l'Allier par les Hotus, et c'est sans doute cet envahissement qui eausa la disparition presque complète de l'Ombre commun.

Ce fut en 1928 que les premiers Hotus apparurent à Brioude. Dans l'enquête piscicole de 1936, ceux-ci y étaient encore peu fréquents (au même titre que la Carpe et la Tanche), et ils étaient estimés constituer $5 \%$ du peuplement de l'Allier dans sa traversée du Puy-de-Dôme.

Maintenant, et depuis une dizaine d'années déjà, ils constituent $70 \%$ du peuplement à l'aval de Brioude, et ils remontent jusqu'à Langeac suivant une proportion décroissante de 50 à $10 \%$.

Sans doute, à l'aval de Brioude, les étés chauds et secs, tel celui de 1962, peuvent à eux seuls faire périr les géniteurs Saumons et les jeunes tacons, faute d'une teneur suffisante d'oxygène dans une eau dont la température atteint et dépasse $25^{\circ} \mathrm{C}$. Mais il est certain que, lors que les conditions climatiques sont favorables à la reproduction des Saumons dans ce très grand secteur, celle-ci n'en est pas moins très sérieusement compromise par la pullulation des Hotus. Or les trois dernières remontées de Saumons (1960, 1961 et 1962) ont à peine (lepassé Brioude et leurs grosses majorités ont même frayé dans le Puy-de-Dôme !

\section{Estimations des zones de frayères actuelles}

C'est seulement dans le bassin de l'Allier qu'il subsiste des zones de bonnes frayères fréquentées par les Saumons et capables, de ce fait, de produire des tacons. Mais des 760 ha que celles-ci couvraient avant la Rérolution, il ne reste plus que :

$1^{\circ} 175$ ha dans l'Allier lui-même, de Brioude à Monistrol d'Allier;

$2^{\circ} 20$ ha dans la Dore, de la restitution de la chute de Sauviat, à Courpierre, et qui, étant les plus en aval, sont d'une production très aléatoire.

Cette estimation, qui totalise donc 200 ha, correspond au $1 / 11$ de l'estimation de ces surfaces d'cau avant la Révolution, et au $1 / 3$ de celle-ci au début de ce siècle.

Lt ce résidu a failli encore être annihilé par l'édification d'un nouveau barrage à Vichy, puis par la modernisation du vieux barrage de VieilleBrioude.

Cependant, l'administration des Eaux et Forèts a été alertéc suffi- 
samment tòt pour pourvoir obtenir dans chacun de ces deux ouvrages deux échelles à poissons à gros débit et bien situées, si bien que l'on peut espérer le maintien des remontées actuelles, qui peuvent être estimées de 1.000 à 3.000 têtes dans l'axe Loire-Allier suivant les années.

Il y a lieu de remarquer que ces restiges de remontées de Saumons correspondent sensiblement au 1/4 des remontées au début du siècle et au $1 / 10$ de celles des environs de 1890, alors que l'estimation ci-dessus des surfaces de frayères encore utilisées représente le $1 / 3$ et le $1 / 5$ de ces surfaces aux mèmes époques.

Nul doute donc, que les zones de frayères actuelles ont, à surface égale, une production de tacons nettement inférieure à celle de jadis (5 à 15 Saumons par hectare de frayères au lieu de 10 à 30).

Cette sous production des zones à frayères subsistantes s'explique facilement du fait de leur envahissement par les Hotus à leur aval, et et par suite des variations brusques des débits de l'Allier dûes aux éclusées de l'usine de Monistrol, qui se font encore très puissamment sentir au moins jusqu'à Langeac. 